\title{
EQUIPE DE SAÚDE DA FAMÍLIA E O USO DE DROGAS ENTRE ADOLESCENTES*
}

Simone Campos Ferreira ${ }^{1}$, Richardson Miranda Machado ${ }^{2}$

RESUMO: Estudo transversal de caráter epidemiológico e descritivo com o objetivo de analisar o uso de álcool e/ou drogas por adolescentes da área de abrangência de uma equipe da Estratégia Saúde da Família, em 2011. Foram entrevistados 142 adolescentes, os quais responderam a dois questionários, um com dados socioeconômicos e outro denominado Drug Use Screening Inventory. Encontrou-se semelhança entre os sexos; maior consumo de drogas entre 10 a 12 anos e 16 a 18 anos de idade; e menor consumo entre os católicos e evangélicos. As substâncias mais consumidas foram analgésico, álcool e tabaco; observou-se maior intensidade de problemas nas áreas de lazer e recreação, competência social e padrões de comportamento. Os dados apresentam informações relevantes para o planejamento de estratégias preventivas ao uso de drogas na adolescência. DESCRITORES: Adolescente; Detecção do abuso de substâncias; Saúde pública.

\section{THE FAMILY HEALTH TEAM AND THE USE OF DRUGS AMONG ADOLESCENTS*}

ABSTRACT: This cross-sectional study, with an epidemiological and descriptive character, aimed to analyze the use of alcohol and/or drugs by adolescents in the territory of a Family Health Strategy team in 2011. 142 adolescents were interviewed, responding to two questionnaires, one with socio-economic data, and the other titled Drug Use Screening Inventory. Similarity was found between the sexes; higher consumption of drugs between the ages of 10 and 12 and 16 and 18 ; and lower consumption among Roman Catholics and Pentecostalists. The most-consumed substances were analgesics, alcohol and tobacco; greater intensity of problems was observed in the areas of leisure and recreation, social competence, and patterns of behavior. The data presents information relevant to the planning of strategies for preventing drug use in adolescence. DESCRIPTORS: Adolescent; Detection of substance abuse; Public health.

\section{EQUIPO DE SALUD DE LA FAMILIA Y EL USO DE DROGAS ENTRE ADOLESCENTES*}

RESUMEN: Estudio transversal de carácter epidemiológico y descriptivo con el objetivo de analizar el uso de alcohol y/o drogas por adolescentes del área de actuación de un equipo da Estrategia Salud de la Familia, en 2011. Fueron entrevistados 142 adolescentes, los cuales contestaron a dos cuestionarios, un con datos socioeconómicos y otro denominado Drug Use Screening Inventory. Se concluyó que hay semejanzas entre los sexos; mayor consumo de drogas entre 10 y 12 años y 16 y 18 años de edad; y menor consumo entre los católicos y evangélicos. Las sustancias más consumidas fueron analgésico, alcohol y tabaco; y se observó mayor intensidad de problemas en las áreas de ocio y recreación, competencia social y tipos de comportamiento. Los datos presentan informaciones relevantes para el planeamiento de estrategias preventivas al uso de drogas en la adolescencia. DESCRIPTORES: Adolescente; Detección del abuso de sustancias; Salud pública.

\footnotetext{
* Extraído do trabalho de conclusão do Curso de Especialização/Residência em Enfermagem na Atenção Básica - Saúde da Família da Universidade Federal de São João Del Rei, Campus Centro Oeste Dona Lindu, em 2011.

${ }^{1}$ Enfermeira. Especialista em Saúde da Família.

${ }^{2}$ Enfermeiro. Doutor em Psiquiatria. Professor do Curso de Graduação e do Curso de Especialização em Atenção Básica - Saúde da Família da Universidade Federal de São João Del Rei.
}

Autor correspondente:

Richardson Miranda Machado

Universidade Federal de Minas Gerais

Rua São Paulo, 1080 - 35500-006 - Divinópolis-MG-Brasil

E-mail: richardson@usp.br
Recebido: $28 / 08 / 2012$ Aprovado: 09/05/2013 


\section{INTRODUÇÃO}

O uso de substâncias psicoativas por adolescentes é considerado um grave problema social e de saúde pública. Estudos sobre o uso de álcool e/ou drogas têm demonstrado que é na adolescência que ocorre o primeiro contato com as drogas pela maioria dos usuários, e que quanto mais cedo se inicia o uso, maior é o risco de se tornar dependente e de sofrer consequências, como acidentes, brigas, conflitos familiares, comportamento sexual de risco, e de cometer suicídio ${ }^{(1-3)}$.

A adolescência é considerada um período de transição física e comportamental. As constantes mudanças do processo de desenvolvimento predispõem os jovens a maior agilidade à situações adversas, tais como influência de amigos, busca pelo desconhecido, curiosidade, fuga das dificuldades pessoais, contradição dos valores estabelecidos pelos familiares, entre outros ${ }^{(4)}$; o que torna o adolescente mais vulnerável ao uso de álcool e/ou drogas e à prática de outros delitos.

Pesquisas tem registrado grande consumo de drogas lícitas pelos adolescentes, sendo a prevalência do uso de bebidas alcoólicas de $65,2 \%$ com dependência de $6,7 \%$ e do uso de tabaco em $16,6 \%$. Esse fator é considerado predisponente para o uso de drogas ilícitas, pois o efeito de substâncias psicotrópicas em um organismo em desenvolvimento leva à dependência e consequente busca por novas substâncias e efeitos ${ }^{(2-3,5-6)}$.

No Brasil, apesar da venda de álcool e tabaco para menores de 18 anos ser proibida, o que se observa é uma conduta social permissiva e omissiva quanto ao cumprimento da lei, principalmente em festas familiares e ocasiões sociais. Isso facilita $o$ acesso dos adolescentes às substâncias psicotrópicas e consequente dependência.

A busca por algo novo e a curiosidade decorrente do ciclo de vida da adolescência leva os jovens a experimentarem diferentes substâncias psicoativas. No Brasil, após o álcool e o tabaco, as drogas com maior frequência de uso pelos adolescentes são a maconha $(8,8 \%)$, solventes $(6,1 \%)$, benzodiazepínicos $(5,6 \%)$ e estimulantes $(3,2 \%)^{(7)}$.

Autores afirmam que o afastamento dos jovens das drogas e de suas complicações deve-se, principalmente, aos valores morais que recebem dos pais; da convivência em um lar harmônico; do não uso de substâncias psicotrópicas pelos familiares; das informações sobre as drogas e os malefícios do seu uso; do acesso às atividades escolares e de lazer; da despreocupação de terem que dar apoio financeiro à família por meio do trabalho precoce, entre outros ${ }^{(7-8)}$.
Conhecidas as razões do não-uso de drogas por adolescentes, torna-se relevante a elaboração de programas de investigação e de prevenção que abordem os diferentes contextos sociais e da saúde pública brasileira. Neste contexto, vale ressaltar que os serviços de atenção básica a saúde, principalmente as Equipes da Estratégia Saúde da Família (ESF) as quais atuam diretamente com o cuidado em todas as fases do ciclo de vida ${ }^{(9)}$, tem papel importantíssimo no rastreamento e prevenção do uso de álcool e/ou drogas pelos adolescentes.

Diante desta realidade, a ESF apresenta-se como importante instrumento de promoção da saúde de adolescentes e de prevenção de agravos como a dependência de álcool e/ou drogas. Por estar inserida e atuar no cenário de vida da população, tornando-se possível conhecer e acompanhar os indivíduos no seu próprio cenário de vida. Assim, sendo capaz de intervir precocemente frente a possíveis fatores de risco e estabelecer medidas de prevenção.

Deste modo, esta pesquisa teve como propósito analisar o uso de álcool e/ou drogas por adolescentes da área de abrangência de uma ESF e cujos resultados podem subsidiar ações de saúde dirigidas para a prevenção do uso dessas substâncias e de problemas associados.

\section{MÉTODO}

Trata-se de estudo transversal de caráter epidemiológico e descritivo ${ }^{(10)}$, realizado em $2011 \mathrm{com}$ os adolescentes da área de abrangência de uma equipe de ESF do Município de Divinópolis-Minas Gerais. $O$ projeto foi apresentado à equipe da ESF e da escola de sua área de abrangência. Foram convidados para participar da pesquisa adolescentes de 10 a 19 anos $(\mathrm{n}=160)$. Foram adotados como critérios de inclusão: ser morador da área de cobertura da ESF, aceitar participar da pesquisa, preencher e assinar o Termo de Consentimento Livre e Esclarecido (TCLE). E tendo como critérios de exclusão o preenchimento errôneo ou incompleto do TCLE e/ou do questionário utilizado.

Na primeira etapa foi realizado um levantamento de todos os adolescentes moradores da área, após foram realizadas visitas domiciliares a fim de convidar e apresentar os projetos aos adolescentes e seus responsáveis legais, em dia e hora pré-agendados. Neste momento foi solicitada a assinatura do TCLE pelos responsáveis e adolescentes.

Para a coleta de dados foram utilizados dois questionários, um elaborado pelos autores da pesquisa para 
abordar os dados sociodemográficos (sexo, idade, religião, raça e presença parietal) e o outro denominado Drug Use Screening Inventory (DUSI), desenvolvido nos Estados Unidos e adaptado à população brasileira (Inventário de Triagem do Uso de Drogas) ${ }^{(11)}$. Essa versão foi utilizada por ser direcionada especialmente para a população adolescente, ser de domínio público e disponibilizada gratuitamente.

A aplicação dos instrumentos de coleta de dados foi realizada na escola localizada na área de abrangência da ESF, em local privativo e somente com a presença dos adolescentes e pesquisadores responsáveis. A média de tempo utilizada foi de 30 a 40 minutos, após o preenchimento os questionários, sem identificação foram depositados em uma urna fechada pelos próprios respondentes.

Para a análise dos dados sociodemográficos foi utilizado a estatística descritiva, para a análise das variáveis do questionário DUSI foram utilizados os escores de densidade absoluta e relativa de problemas.
O trabalho seguiu todas as salvaguardas estabelecidas pelo Comitê de Ética em Pesquisa e foi aprovado sob o parecer n. 106/2011. E obedeceu aos termos da Resolução n. 196/96 do Conselho Nacional de Ética em Pesquisa envolvendo seres humanos.

\section{RESULTADOS}

Do total de 160 adolescentes da área de abrangência, 142 participaram da pesquisa, totalizando $88,75 \%$. No que se refere aos dados socidemográficos dos adolescentes participantes os mesmos encontram-se apresentados na tabela 1. Houve distribuição semelhante em relação ao sexo; à faixa etária predominante foi entre 13 e 15 anos. A maioria se autodenominou católico, seguidos de evangélicos. Ao comparar o uso de drogas com a religião, foi encontrado que $19(63,3 \%)$ adolescentes que se declaram ser de outra religião faziam uso de drogas, seguidos de 45(57,6\%) católicos e 17(50\%) espíritas.

Tabela 1 - Dados sociodemográficos dos adolescentes da área de abrangência de uma equipe de saúde da família do município de Divinópolis, 2011

\begin{tabular}{llcc}
\hline Variáveis & Característica dos adolescentes & n & $\%$ \\
\hline \multirow{2}{*}{ Sexo } & Feminino & 72 & 50,7 \\
& Masculino & 70 & 49,3 \\
\multirow{2}{*}{ Idade } & 10 a 12 anos & 57 & 40,1 \\
& 13 a 15 anos & 72 & 50,7 \\
& 16 a 18 anos & 13 & 9,1 \\
Religião & Católico & 78 & 54,9 \\
& Evangélico & 34 & 23,9 \\
& Outra & 30 & 21,1 \\
Raça & Branca & 33 & 23,2 \\
& Negra & 16 & 11,2 \\
& Mulata/Morena & 88 & 61,9 \\
\multirow{2}{*}{ Presença dos pais } & 5 & 3,5 \\
& Amarela/Ocidental & 88 & 61,9 \\
& Pai e mãe & 45 & 31,7 \\
& Apenas da mãe & 5 & 3,5 \\
& Apenas do pai & 4 & 2,8 \\
\hline
\end{tabular}

No que tange a raça, a maioria dos jovens se definiu como da raça mulata/morena. Ao se comparar a raça com uso de drogas foi verificado um maior consumo pela raça negra, com $11(68,7 \%)$ respondentes, seguida da raça amarela/ocidental 3(60\%), branca 19(57,5\%) e mulata/morena $48(54,5 \%)$. Quanto à presença parietal $88(61,9 \%)$ adolescentes relataram viver com o pai e a mãe e $45(31,7 \%)$ viviam somente com a mãe.
Ao se avaliar o uso de álcool e/ou drogas foi possível identificar a predominância do uso de analgésico, álcool e tabaco, de acordo com dados da tabela 2. Em relação ao sexo não foi obsevado diferença significativa de consumo. Foi observado que a maioria dos adolescentes que consumiam alguma substância se encontrava na faixa etária de 10 a 12 anos, seguido da faixa etária de 16 a 18 anos. Ressalta-se que 61(42,9\%) 
adolescentes relataram que nunca consumiram droga.

Ao analisar as áreas do questionário DUSI, foi possível identificar o perfil da intensidade de problemas em relação ao uso de drogas. No que se refere à densidade absoluta e relativa de problemas em cada área da vida dos adolescentes, os resultados encontram-se apresentados na tabela 3 .

Ao se estabelecer a densidade absoluta de pro- blemas, a qual indica a intensidade de problemas em cada área da vida do adolescente isoladamente, verificou-se maior intensidade de problemas nas áreas de lazer e recreação, competência social e padrões de comportamento. Assim como, a densidade relativa de problemas, que indica a contribuição percentual de cada área no total de problemas, também detectou-se as mesmas áreas.

Tabela 2- Uso de álcool e outras drogas por sexo e idade dos adolescentes da área de abrangência de uma Equipe de Saúde da Família, segundo o Drug Use Screening Inventory e questionário sóciodemográfico. Divinópolis, 2011

\begin{tabular}{lccccc}
\hline Variávies & $\begin{array}{c}\text { Feminino } \\
\mathbf{n}(\mathbf{\%})\end{array}$ & $\begin{array}{c}\text { Masculino } \\
\mathbf{n ~ ( \% )}\end{array}$ & $\begin{array}{c}\mathbf{1 0} \text { a 12 anos } \\
\mathbf{n}(\mathbf{\%})\end{array}$ & $\begin{array}{c}\mathbf{1 3} \text { a 15 anos } \\
\mathbf{n ~ ( \% )}\end{array}$ & $\begin{array}{c}\mathbf{1 6} \text { a 18 anos } \\
\text { n (\%) }\end{array}$ \\
\hline Álcool & $20(27,7)$ & $24(34,2)$ & $17(29,8)$ & $19(35,8)$ & $8(61,5)$ \\
Anfetaminas/ & - & $1(1,4)$ & - & $1(1,8)$ & - \\
Estimulantes & - & $2(2,8)$ & - & $1(1,8)$ & $1(7,6)$ \\
Extase & - & $3(4,2)$ & $1(1,7)$ & $1(1,8)$ & $1(7,6)$ \\
Cocaína/Crack & - & $9(12,8)$ & $2(3,5)$ & $6(11,3)$ & $2(15,3)$ \\
Maconha & $1(1,3)$ & $1(1,4)$ & - & $1(1,8)$ & - \\
Alucinógenos & - & $4(5,7)$ & $4(7,0)$ & $3(5,6)$ & - \\
Tranquilizantes & $3(4,1)$ & $23(32,8)$ & $25(43,8)$ & $21(39,6)$ & $3(23,0)$ \\
Analgésicos & $26(36,1)$ & $1(1,4)$ & - & $1(1,8)$ & - \\
Opiáceos & - & $1(1,4)$ & - & $1(1,8)$ & - \\
Fenilciclidina & - & $4(5,7)$ & $1(1,7)$ & $3(5,6)$ & - \\
Anabolizantes & - & $2(2,8)$ & $1(1,7)$ & $2(3,7)$ & - \\
Inalantes/ & $1(1,3)$ & $13(18,57)$ & $5(8,77)$ & $12(22,64)$ & $2(15,38)$ \\
solventes & $6(36,11)$ & $2(2,8)$ & - & $1(1,8)$ & $1(7,6)$ \\
Tabaco & - & & & & \\
Outros & & & &
\end{tabular}

Tabela 3- Perfil da intensidade de problemas em relação ao uso de drogas pelos adolescentes da área de abrangência de uma Equipe de Saúde da Família, segundo o Drug Use Screening Inventory. Divinópolis, 2011

\begin{tabular}{lcccc}
\hline Áreas & Pontuação total (n) & Pontuação bruta & $\begin{array}{l}\text { Densidade absoluta Densidade relativa } \\
\text { de problemas (\%) }\end{array}$ & $\begin{array}{l}\text { De problemas (\%) } \\
\text { de }\end{array}$ \\
\hline $\begin{array}{l}\text { Comportamento de } \\
\text { uso de substâncias }\end{array}$ & 2130 & 163 & 7,6 & 2,9 \\
$\begin{array}{l}\text { Padrões de compor- } \\
\text { tamento }\end{array}$ & 2840 & & & \\
Área da saúde & 1420 & 902 & 31,7 & 12,3 \\
Desordem & 2840 & 383 & 26,9 & 10,4 \\
psiquiátrica & 1988 & 864 & 30,4 & 11,8 \\
Competência social & 1988 & 667 & 33,5 & 13,0 \\
Sistema Familiar & 2840 & 602 & 30,8 & 11,9 \\
Escola & 1420 & 760 & 26,7 & 10,3 \\
Trabalho & 96 & 6,7 & 2,6 \\
Relacionamento com & 1988 & 574 & 28,8 & 11,2 \\
colegas & 1704 & 581 & 34,09 & 13,23 \\
Lazer e recreação & 21158 & 5592 & 257,6 & 99,9 \\
Total & & & & \\
\hline
\end{tabular}




\section{DISCUSSÃO}

Em relação ao gênero houve participação semelhante do número de adolescentes do sexo masculino e feminino, tal fato coincide com os dados obtidos no Sistema de Informação da Atenção Básica (SIAB) ${ }^{(12)}$ da referida ESF trabalhada, a qual mostra não haver grande variação de gênero na área.

Estudos apontam o maior uso de drogas por adolescentes do sexo masculino, diferente do detectado por este estudo, no qual houve semelhança do consumo de drogas entre os sexos. $O$ fato de a mulher estar cada vez mais conquistando espaço na sociedade e tomando atitudes que antes eram tidas pelo homem pode ser uma das razões para o aumento do uso de drogas pelo gênero feminino ${ }^{(1,13-14)}$, levando à equiparação entre gêneros.

As mudanças sociais decorrentes das conquistas femininas fizeram com que a mulher passasse a ser mais valorizada, reduzindo o estigma de que mulher só serve para cuidar da casa e dos filhos. Hoje a mulher disputa o mercado de trabalho, divide as funções domésticas com os homens, ajuda nas finanças, possui sua independência financeira. Por outro lado, a tensão gerada por exercer diversas funções, o estresse, a ansiedade e a tentativa em parear-se em tudo com o sexo oposto, pode levá-las para o mundo das drogas.

Foi observado maior consumo de drogas nas faixas etárias de 10 a 12 anos e de 16 a 18 anos, semelhante ao encontrado em outros estudos ${ }^{(3,15)}$, os quais trazem, como possíveis motivos, o início da puberdade ou o alcance da maior idade judicial, pertencer à classes sociais economicamente desfavorecidas, disfunção familiar e curiosidade. Os jovens querem adentrar no meio social e afirmar sua identidade; a falta de acesso ao lazer, cultura, trabalho e problemas familiares predispõem os adolescentes a conflitos íntimos e ao uso de drogas.

A inserção do jovem no meio social deve se estimulada desde a infância com a família, passando pela escola e relação com os amigos. Para que este processo aconteça de forma segura e saudável os jovens devem receber apoio familiar, incentivo para a educação e trabalho, bem como estímulo para a prática de esportes. $\mathrm{O}$ aprendizado dos jovens, através dos adultos de referência e grupos de convivência, tem impacto positivo quanto a prática de hábitos saudáveis de vida ${ }^{(16)}$.

Ao se analisar a religião, observou-se menor consumo de drogas pelos adolescentes que se autodenominaram católicos e evangélicos, quando comparado com os adolescentes de outras religiões. As religiões mais conservadoras tendem a apresentar menor número de consumidores de drogas; quanto maior a frequência religiosa menor o uso ${ }^{(17)}$. Estudosconfirmam que ter uma religião é considerado um fator de proteção para uso de drogas $^{(18-21)}$. Deste modo, estimular o envolvimento dos adolescentes com igrejas e/ou associações religiosas é um fator de proteção para uso de álcool e outras drogas por ser considerados locais de socialização, além de auxiliar na construção da personalidade do indivíduo e incentivar a preservação da vida.

Na avaliação quanto a raça dos adolescentes entrevistados, a maioria classificou como sendo da raça morena/ mulata; sendo esta uma característica observada entre a população da área de abragência do estudo. Apesar de estar em menor número, o consumo de drogas prevaleceu entre os adolescentes de raça negra. A herança histórica de exclusão social sofrida pela raça negra ainda resulta na falta de oportunidade de estudo e emprego, o que predispõe esta população adentrar no mundo das drogas.

Ao se abordar a presença parietal, a maioria dos estudantes vivia com ambos os pais. Estudos mostram que grande parte dos adolescentes que não fazem uso de drogas tem este tipo de composição familiar, demonstrando que a estrutura da família tem um papel preponderante no comportamento e na qualidade de vida dos adolescentes. A família é considerada o primeiro contato do indivíduo com o meio social, portanto a boa convivência familiar, o afeto e a adequada conduta social dos pais são tidos como fatores de proteção para o não uso de substâncias psicotrópicas $^{(3,16)}$.

Pesquisa demonstra que a composição monoparental, ou seja, só a mãe ou o pai presente, predomina entre os adolescentes usuários de drogas. Esta estrutura familiar favorece o uso de drogas uma vez que apresenta maior probabilidade para a permissividade em detrimento da autoridade parietal dividida, ausência de limites claros e/ou a falta de normas e regras de comportamento, contribuindo para o adolescente não encontre adequadas manifestações de afeto e de conduta social ${ }^{(22)}$.

Autores também afirmam que ter pais violentos, abuso de poder, violência física e psicológica, podem ser mais prejudiciais do que a ausência de um ou de ambos os pais. Estes aspectos levam o jovem a se refugiar nas drogas, com intuito de esquecer estes conflitos ${ }^{(2,4,23-24)}$.

No que se refere ao uso de drogas, a mais utilizada pelos adolescentes foi o analgésico, o que pode estar relacionado ao fato deste medicamento ser de fácil acesso nas farmácias, pois não necessita de receita médica para ser adquirido, estar disponível nas unidades de saúde, nos domicílios e escolas. Estudo realizado identificou o uso de analgésicos por aproximadamente $60 \%$ dos adolescentes, 
tendo como principal motivo para o uso o acometimento por cefaleias, cólicas menstruais e dores musculares, queixas comuns entre os adolescentes ${ }^{(21)}$.

Cabe ressaltar que mesmo que o analgésico seja utilizado para intercorrências temporárias de saúde e de ter seu uso habitual pela população, o mesmo é um medicamento e por isso traz riscos para a saúde. Neste sentido, o uso deve ser prescrito por um profissional de saúde e o consumo por jovens, feito sob a supervisão de um adulto.

Foi observado também grande consumo de álcool e tabaco pela população estudada. Tal ocorrência pode ser compreendida pelo fato destas drogas serem utilizadas como meio de socialização, o que estimula o consumo pelos adolescentes para se sentirem adultos, perderem a timidez, se sentirem mais encorajados e mais seguros nos primeiros contatos afetivos e amorosos.

O uso de álcool e tabaco é um problema que afeta não somente o indivíduo, mas também a família e a comunidade, e traz sérias consequências como o desenvolvimento de tolerância. Isso faz com que o indivíduo queira sempre mais e o leva a experimentar outras sensações, com outros tipos de drogas, envolvimento em acidentes automobilísticos e brigas, maior exposição à doenças sexualmente transmissíveis, prejuízos escolares, doenças no fígado, pulmão e cardiovasculares. De acordo com pesquisadoreso uso do álcool é um dos principais fatores associados à redução da saúde mundial, sendo responsável por 3\% de todas as mortes e por $4 \%$ de todos os anos de vida útil perdidos; e o tabaco responsável por $90 \%$ das mortes por problemas respiratórios ${ }^{(25)}$.

Neste estudo foi observado também o uso de outras substâncias, como maconha, crack/cocaína, anabolizantes, entre outras. A conscientização dos jovens a respeito da prevenção para o uso de droga seja, lícita ou ilícita, e a conscientização sobre os problemas por elas causados, se torna importante para a não experimentação destas. $\mathrm{O}$ diálogo entre a família, a abordagem deste assunto nas escolas e unidades de saúde favorecem a prevenção do uso entre os adolescentes ${ }^{(2,26)}$.

Ao se analisar a intensidade de problemas em cada área da vida dos adolescentes, identificou-se que as áreas com maior intensidade de problemas foram as de lazer e recreação, competência social e padrões de comportamento. Estes dados reiteram o fato de que a área de abragência da ESF é desprovida de locais de lazer e integração social.

A falta de acesso à recreação, cursos profissionalizantes e locais para a prática de esportes, faz com que os jovens fiquem ociosos no período de contraturno da escola, o que os predispõem aos uso de drogas. Torna- -se, portanto necessário o desenvolvimento de políticas públicas voltadas para esta população, que contribua para a evitar ou retardar a experimentação e, diminuir o uso esporádico de drogas entre os jovens. As ações, principalmente educativas, devem ser desenvolvidas em parceria com as escolas e ESF, além de serem oferecidas alternativas esportivas e culturais para os adolescentes.

Cabe ressaltar que a competência social se refere ao conjunto de comportamentos aprendidos nas interações com os outros (família, amigos, escola), que abarca variabilidade de habilidades sociais e aptidões, as quais mostram ter um papel essencial no desenvolvimento dos adolescentes. Estudos confirmam que boas habilidades de competência social estão relacionadas com a diminuição da delinquência, agressão, consumo de drogas e outros problemas de conduta apresentados por adolescentes ${ }^{(5,22)}$.

Diante dos resultados deste estudo torna-se necessário o estabelecimento de vínculo entre a escola e a ESF, para que seja possível o desenvolvimento de atividades de prevenção do uso de drogas, tais como palestras para adolescentes e familiares, formação de grupos de jovens, dinâmicas, campeonatos e oficinas de trabalho.

Vale ressaltar que a ESF, entendida como uma proposta de ação, busca a responsabilização pela saúde da população territorializada. Desse modo, as equipes desse âmbito assistencial devem ampliar a prática curativa e preventiva do modelo biomédico tradicional, a fim de promover a qualidade de vida e a prevenção de agravos.

\section{CONSIDERAÇÕES FINAIS}

O estudo mostrou que o uso de drogas pelos adolescentes acontece precocemente e que este é semelhante entre os sexos. Diversos são os fatores que pode contribuir para o uso, como composição familiar, falta de acesso a lugares de lazer e recreação, ausência de religião.

As drogas mais consumidas entre os adolescentes da área de abrangência foram as lícitas, sendo estas consideradas a porta de entrada para o consumo de substâncias ilícitas. Desta forma, torna-se necessário uma maior fiscalização e conscientização dos proprietários de estabelecimentos comerciais sobre a proibição da venda de bebidas alcoólicas para menores de 18 anos, bem como a participação dos familiares na prevenção do uso.

A promoção da parceria entre escola e ESF é importante para que o estabelecimento de ações de prevenção do uso de álcool e/ou drogas pelos adolescentes da área de abrangência, levando em conta os resultados do presente estudo.

A utilização do questionário DUSI nos serviços de saúde e escola pode facilitar intervenções e práticas 
preventivas com os adolescentes. Constitui instrumento que facilita a abordagem e auxilia na condução de intervenções com base nas áreas de maior incidência de problemas.

\section{REFERÊNCIAS}

1. Garcia JJ, Pillon SC, Santos MA. Relações entre contexto familiar e uso de drogas em adolescentes de ensino médio. Rev. Latino-Am. Enferm. 2011;19:753-61.

2. Pratta EMM, Santos MA. Família e adolescência: a influência do contexto familiar no desenvolvimento psicológico de seus membros. Psicol. estud. 2007;12(2):247-56.

3. Campos JADB, Almeida JC, Garcia PPNS, Faria JB. Consumo de álcool entre estudantes do ensino médio do município de Passos/MG. Ciênc. saúde colet. 2011;16(12):4745-54.

4. Lima IS, Paliarin MM, Zaleski EGF, Arantes SL. História oral de vida de adolescentes dependentes químicos, internados no setor de psiquiatria do hospital regional de Mato Grosso do Sul para tratamento de desintoxicação. SMAD. 2008;4(1):1-11.

5. Jinez LJ, Souza JRM, Pillon SC. Uso de drogas e fatores de risco entre estudantes de ensino médio. Rev. LatinoAm. Enferm. 2009;17(2):246-52.

6. Silveira CM, Silveira CS, Silva JG, Silveira LM, Andrad AG, Andrad LHSG. Epidemiologia do beber pesado e beber pesado episódico no Brasil: uma revisão sistemática da literatura. Rev. psiquiatr. clín. 2009;35(1):31-8.

7. Carlini EA, Galduróz JCF, Noto AR, Fonseca AM, Carlini CM, Oliveira LG, et al. II Levantamento Domiciliar sobre o uso de drogas psicotrópicas no Brasil: estudo envolvendo as 108 maiores cidades do país: 2005 - São Paulo: CEBRID São Paulo: Páginas \& Letras; 2007.

8. Wagner GA, Andrade AG. Uso de álcool, tabaco e outras drogas entre estudantes universitários brasileiros. Rev. psiquiatr. clín. 2008;35(1):48-54.

9. Ministério da Saúde (BR). Programas e Projetos - Saúde da família [Internet]. Brasília; 1998. [acesso em $16 \mathrm{dez}$ 2011] Disponível: http://portal.saude.gov.br/saude

10. Gordis L. Epidemiologia. Rio de Janeiro: Revinter; 2004.
11. De Michelli D, Formigoni MLOS. Screening of drung use in the a teenage Brazillian sample using the Drug Use Screening Inventory (DUSI) Addict. behav. 2000;25(5):683-91.

12. Minas Gerais. Secretaria Municipal de Saúde (SEMUSA). Sistema de Informação da Atenção Básica/2011. Divinópolis; 2012.

13. Galduróz JCF, Noto AR, Fonseca AM, Carlini EA. V Levantamento Nacional sobre o consumo de drogas psicotrópicas entre estudantes do ensino fundamental e médio da rede pública de ensino nas 27 capitais brasileiras - 2004. São Paulo: Centro Brasileiro de Informações sobre Drogas Psicotrópicas/Departamento de Psicobiologia - UNIFESP; 2005.

14. Silva EA, De Micheli D, Camargo BMV, Buscatti D, Alencar MAP, Formigoni MLOS. Drogas na adolescência: temores e reações dos pais. Psicol. teor. prat.2006;8(1):41-54.

15. Ortega-Perez CA, Costa-Junior ML, Vasters GP. Perfil epidemiológico da toxicodependência em estudantes universitários. Rev. Latino-Am. Enfermagem. 2011;19(n. esp):665-72.

16. Costa COM, Alves MVQM, Santos CAST, Souza KEP, Sousa HL. Experimentação e uso regular de bebidas alcoólicas, cigarros e outras substâncias psicoativas (SPA) na adolescência. Ciênc. saúde colet. 2007;12(5):1143-54.

17. Sanchez ZM, Nappo AS. A religiosidade, a espiritualidade e o consumo de drogas. Rev. psiquiatr. clín. 2007;34(1):73-81.

18. Pillon SC, Santos MA, Gonçalves MAS, Araújo KM. Uso de álcool e espiritualidade entre estudantes de enfermagem. Rev Esc Enferm USP 2011;45(1):100-7.

19. Silva IM, Catrib AMF, Matos VC, Gondim APS. Automedicação na adolescência: um desafio para a educação em saúde. Ciênc. saúde colet. 2011;16(1):1651-60.

20. Siqueira DF, Moreschi C, Backes DS, Lunardi VL, Filho WDL, Dalcin CB. Repercussões do uso de crack no cotidiano família. Cogitare enferm. 2012;17(2):248-54

21. Alves DS, Lacerda JSJ, Matias TC, Almeida JM, Brito BG, Borlini PG, et al. Estudo do uso de analgésico por crianças e adolescentes de uma escola pública. Rev. bras. pesq. saúde. 2011;13(3):36-42.

22. Garcia JJ, Pillon SC, Santos MA. Relações entre 
contexto familiar e uso de drogas em adolescentes de ensino médio. Rev. Latino-Am. Enferm. 2011;19:753-61.

23. Oliveira EB, Bittencourt LP, Carmo AC. A importância da família na prevenção do uso de drogas entre crianças e adolescentes: papel materno. SMAD. 2008;4(2):1-16.

24. Dietz G, Santos CG, Hildebrandt LM, Leite MT. As relações interpessoais e o consumo de drogas por adolescentes. SMAD. 2011;7(2):85-91

25. Sena ELS, Boery RNSO, Carvalho PAL, Reis HFT, Marques AMN. Alcoolismo no contexto familiar: um olhar fenomenológico. Texto Contexto Enferm. 2011;20(2):310-8.

26. Malbergier A, Cardoso LRD, Amaral RA. Uso de substâncias na adolescência e problemas familiares. Cad. Saúde Pública. 2012;28(4):678-88. 